\title{
BMJ Open Using the behaviour change technique taxonomy v1 (BCTTv1) to identify the active ingredients of pharmacist interventions to improve non- hospitalised patient health outcomes
}

Claire Scott (D) , ${ }^{1}$ Mícheál de Barra (D) , ${ }^{2}$ Marie Johnston, ${ }^{3}$ Marijn de Bruin, ${ }^{3,4}$ Neil Scott, ${ }^{5}$ Catriona Matheson, ${ }^{6}$ Christine Bond, ${ }^{7}$ Margaret C Watson ${ }^{8}$

To cite: Scott C, de Barra M, Johnston M, et al. Using the behaviour change technique taxonomy v1 (BCTTv1) to identify the active ingredients of pharmacist interventions to improve non-hospitalised patient health outcomes. BMJ Open 2020;10:e036500. doi:10.1136/ bmjopen-2019-036500

- Prepublication history for this paper is available online. To view these files, please visit the journal online (http://dx.doi. org/10.1136/bmjopen-2019036500).

Received 06 January 2020 Revised 15 July 2020

Accepted 14 August 2020
Check for updates

(C) Author(s) (or their employer(s)) 2020. Re-use permitted under CC BY-NC. No commercial re-use. See rights and permissions. Published by BMJ.

For numbered affiliations see end of article.

Correspondence to

Claire Scott;

claire.scott@nes.scot.nhs.uk

\section{ABSTRACT}

Objectives The aim of this study was to identify which behaviour change techniques (BCTs) were present in intervention and control groups of randomised controlled trials (RCTs) included in a Cochrane systematic review. Setting The RCTs included were conducted in community, primary and/or ambulatory-care settings.

Participants The data set was derived from 86 RCTs from an interim update of the Cochrane review of the effectiveness of pharmacist services on non-hospitalised patient outcomes.

Primary and secondary outcome measures The primary outcome was the identification of BCTs scheduled for delivery in intervention and control groups of the RCTs. The secondary outcome measure was to identify which BCTs are not being utilised in intervention and control groups of the RCTs.

Results The intervention and control groups included 31 and 12 BCTs, respectively. The number of identifiable $\mathrm{BCTs}$ /study ranged from 0 to 12 in the intervention groups (mean 3.01 (SD 2.4)) and 0 to 6 in the control groups (mean 0.38 (SD 0.84)). The most commonly identified BCTs in the intervention groups were: instruction on how to perform the behaviour ( $55 \%, \mathrm{n}=47$ ) (also the most common BCT in control groups); problem solving (29\%, $\mathrm{n}=25)$; information about health consequences (24\%, $\mathrm{n}=21)$; social support (practical) $(24 \%, \mathrm{n}=21)$; and social support (unspecified) $(23 \%, n=20)$ (the second most common BCT in control groups). Thirteen trials had no identifiable BCTs in either group.

Conclusion The pharmacist interventions presented in this study did not use the full range of available BCTs. Furthermore, the reporting of BCTs was incomplete for both intervention and control groups, thereby limiting the utility and reproducibility of the interventions. Future interventions should be designed and reported using relevant taxonomies and checklists for example, BCT taxonomy and TIDieR (the template for intervention description and replication).

\section{BACKGROUND}

Behaviour change interventions tend to be complex and consist of many different

\section{Strengths and limitations of this study}

- This study was able to identify behaviour change techniques (BCTs) used, as well as those not used, in randomised controlled trials of pharmacist interventions (and control groups).

- There was a high level of agreement between the coders using the BCT taxonomy v1 (BCTTv1).

- The accuracy of the BCT coding was reliant on the information provided in the studies and supplementary materials, which was not always detailed enough to give an accurate description of each intervention and control group.

- This study helps to emphasise the need for consistent reporting of BCTs in studies of pharmacist interventions (and control groups) to inform future developments.

active ingredients working within the one intervention. ${ }^{1}$ It can therefore be difficult to pinpoint the individual active ingredients that are being used in order to change behaviour. It is vital that these are identifiable for transparency, implementation, literature synthesis and future research. For example, a randomised controlled trial (RCT) to evaluate the effectiveness of a medicine will specify the active ingredient(s) of the test drug, together with the strength, dose, frequency and duration, in order for the evaluation to be comprehensible and replicable. If trial authors simply stated that a white pill was being tested, this information and the trial itself would be useless due to the lack of detailed information. The latter scenario reflects the level of specification of the behaviour change intervention in many trials which evaluate their effectiveness. All the active ingredients of a behaviour change 
intervention should be clearly defined and reported to enable their replication.

The behaviour change technique taxonomy v1 $\left(\right.$ BCTTv1) ${ }^{2}$ was developed to provide a shared, standardised terminology to be used to specify the active ingredients of behaviour change interventions, that is BCTs. ${ }^{3}$ The BCTTv1 is a reliable taxonomy which consists of 93 unique BCTs that are categorised into 16 groups. Each BCT has its own number pertaining to its group and position within that group. BCTs are the 'active ingredients' of a behaviour change intervention. They are observable, replicable and irreducible. ${ }^{4}$ These BCTs are specific, each with their own individual label and definition that can be used when designing and reporting interventions, but also when coding interventions for their content.

Several systematic reviews with meta-regression found that interventions that contain certain BCTs are more effective than interventions without that BCT, for example, action planning to enhance physical activity; self-monitoring and providing instruction for weight loss in obese adults; ${ }^{5}$ selfmonitoring to increase physical activity and healthy eating. ${ }^{6}$

The BCT taxonomy is a promising tool for pharmacist interventions. Intervening to enable clients to change self-management behaviours has become part of the changing role of pharmacists in recent years, as well as more traditional dispensing role. Additional behavioural and clinical activities taken on by pharmacists include medication review, ${ }^{7}$ adherence support ${ }^{8}$ and advising practitioners on their prescribing, ${ }^{9}$ as well as enhanced roles in public health. ${ }^{11}{ }^{11}$ The pharmacist is therefore becoming an ever more important figure in public health, particularly through their implementation of behaviour change interventions.

The impact of pharmacists' non-dispensing roles was reviewed by Nkansah et at in a Cochrane systematic review which found that pharmacist interventions, in general, lead to improved clinical outcomes, therefore supporting the role of pharmacists in behaviour change interventions. However, when that review was published, the BCTTv1 had not been developed and the underpinning behavioural techniques used within interventions were not reported. Investigating which BCTs are used and which are associated with improved outcomes can advance both research and practice in this area by allowing links to theoretical frameworks and by providing additional information about methods that might be implemented in practice to enhance effectiveness.

Furthermore, while the previous review was able to make comparisons between outcomes in intervention and control groups in RCTs, it did not report the support given to participants in the control groups as part of 'standard care' or 'treatment as usual'. Given that the size of effect of a trialled intervention will be affected by the practice in the control group, it is important to attempt to specify any BCTs delivered in both groups. ${ }^{12} 13$

This current study stems from an interim update of the Cochrane systematic review. ${ }^{14}$ The aim of the current study was to identify the BCTs evaluated in the intervention and control groups reported in RCTs included in the systematic review. The primary outcome was to identify the BCTs scheduled for delivery in intervention and control groups of the RCTs. The secondary outcome measure was to identify which BCTs are not being utilised in the intervention and control groups of the RCTs.

\section{METHODS \\ Design and study selection}

The data set for this current study comprised the 86 studies included in the interim update of the Cochrane systematic review. ${ }^{14}$ The studies were RCTs of pharmacist interventions to improve non-hospitalised patient outcomes and were published between October 1979 and January 2015. (The review was further updated in January 2018. This current study is based solely on the 86 studies in the interim version of the review.) BCTs were identified and coded in both intervention and control groups.

\section{Behaviour change technique coding}

Authors of the 86 included studies were contacted where possible via email to request additional materials such as protocols and study materials. Two reviewers (CS and $\mathrm{MdBa}$ ) underwent training in BCT coding using an online training tool. ${ }^{15}$ Coding using BCTTv $1^{2}$ was piloted with five studies taken from the excluded studies of the review to compare and refine the coding process. For all the included studies, the behaviour change intervention in both the intervention and control groups was independently coded by both authors using the BCTTv1. Discrepancies were resolved by discussion and, if necessary, a third trained coder (MJ) made the final decision.

\section{Data analysis}

Inter-rater reliability of the coding was calculated using Cohen's kappa adjusted for prevalence and bias (PABAK) ${ }^{16}$ PABAK is a measure of inter-rater agreement which controls for chance agreement and which is particularly useful in situations where there are high levels of negative agreement as is often found in coding BCTs that is, when both coders agree that a BCT is absent. To assess the primary outcome, the frequency of BCTs used in both the intervention and control groups was calculated and reported. To assess the secondary outcome, BCTs not utilised in either the intervention or control group of any study were identified and reported.

\section{Patient and public involvement}

This research was done without patient involvement. Patients were not invited to comment on the study design and were not consulted to develop patient-relevant outcomes or interpret the results. Patients were not invited to contribute to the writing or editing of this document for readability or accuracy. 


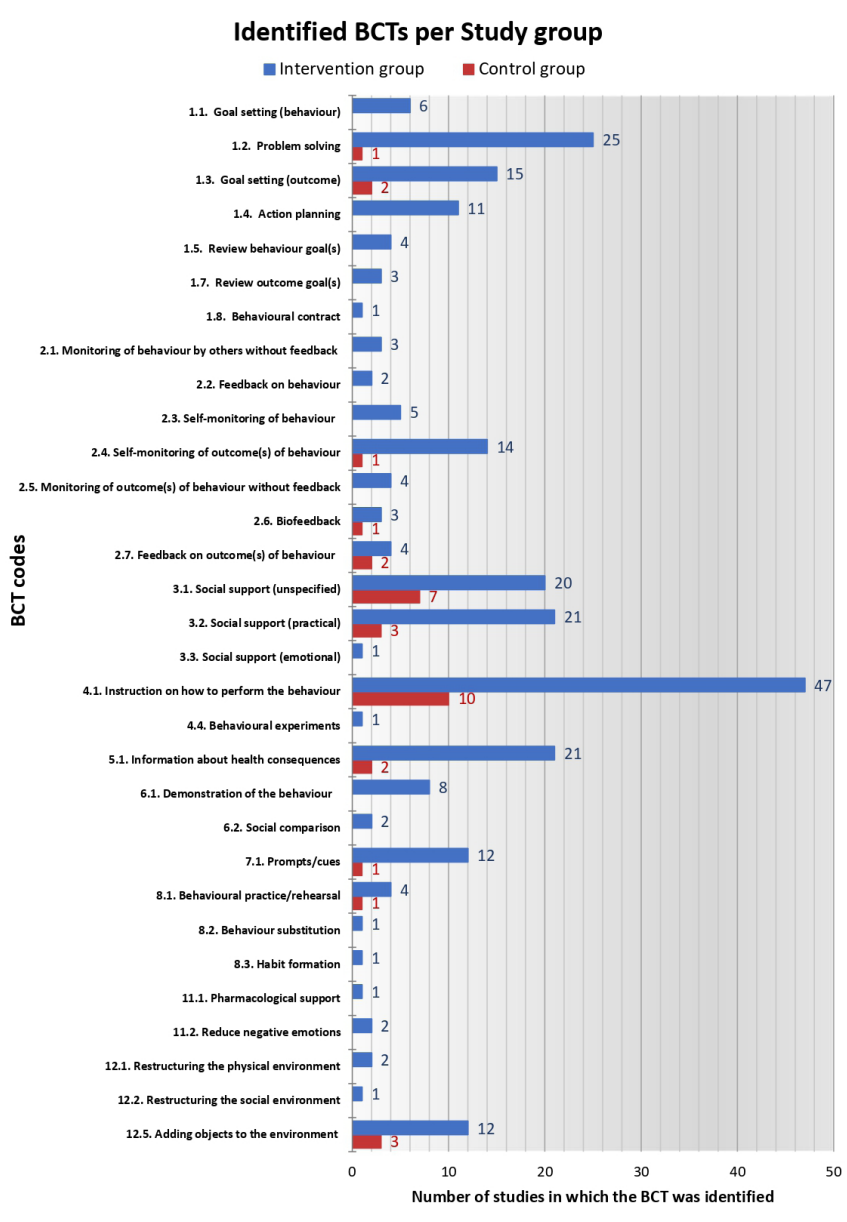

Figure 1 Number of studies reporting each BCT in intervention and control group descriptions. BCT, behaviour change technique.

\section{RESULTS}

An email response was received from authors of 47 studies and relevant responses were received from 32, who provided additional materials associated with the content or delivery of their intervention in both intervention

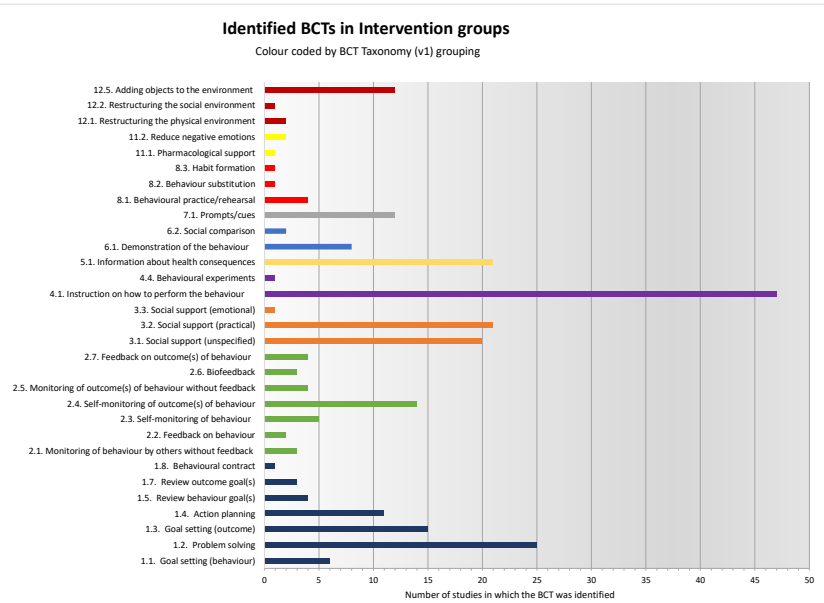

Figure 2 BCTs in intervention group descriptions. BCT, behaviour change technique.

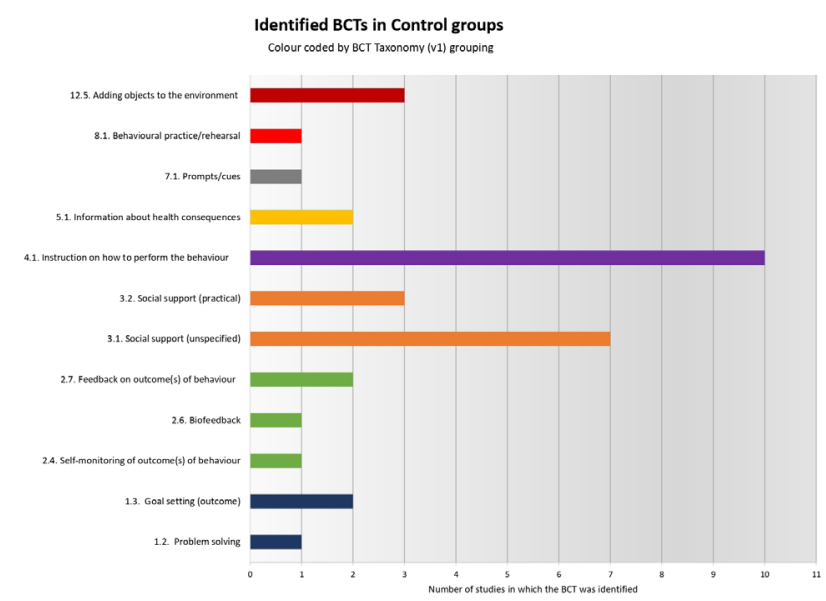

Figure 3 BCTs in control group descriptions. BCT, behaviour change technique.

and/or control groups. The overall inter-rater reliability for BCT coding of all 86 studies was high, PABAK=0.94.

Of the 86 studies coded for BCTs, at least one BCT could be identified and coded in 73 intervention descriptions. Within intervention groups, the number of identifiable BCTs per study ranged from 0 to 12 (median 3 (IQR: 1 to 4), mean 3.01 (SD: 2.4)). In control groups, at least one BCT could be identified and coded in 23 of the 86 studies and the number of identifiable BCTs per study ranged from 0 to 6 , (median 0 (IQR: 0 to 1 ), mean 0.38 (SD: 0.84 )).

\section{Which BCTs were used?}

Thirty-one of the possible 93 BCTs in the BCTTv1 were identified (figure 1). All 31 were used in intervention groups and 12 of these were delivered to control groups. Each BCT was used more often with intervention than with control groups. The most commonly identified BCTs for the intervention groups were: 4.1 instruction on how to perform the behaviour ( $54.7 \%$ of all intervention descriptions included this BCT), 1.2 problem solving (29.1\%), 5.1 information about health consequences (24.4\%), 3.2 social support (practical) $(24.4 \%)$ and 3.1 social support (unspecified) $(23.3 \%)$ (figure 2).

The most commonly identified BCTs in the control groups were: 4.1 instruction on how to perform the behaviour (11.6\% of all control descriptions included this BCT) and 3.1 social support (unspecified) $(8.1 \%$ ) (figure 3 ).

Of the 93 BCTs in the BCTTv1, 62 were not identified in any intervention or control group description. Of the 16 groups of BCTs within the BCTTv1, 10 were identifiable in the coded studies represented in bold table 1 (figures 2 and 3 display the individual BCTs colour coded by their BCT grouping); six groups of BCTs were not represented in any study (table 1).

Thirteen studies had no identifiable BCT in either their intervention or control descriptions. Eight of these RCTs reported a positive intervention effect on at least one of the outcome measures. 
Table 1 BCT groupings $(n=16)$

\section{Groupings in BCTTv1}

\begin{tabular}{ll}
\hline 1. Goals and planning* & 9. Comparison of outcomes \\
2. Feedback and & 10. Reward and threat \\
monitoring
\end{tabular}

\section{Social support ${ }^{\star}$}

11. Regulation*

\section{Shaping knowledge ${ }^{\star}$ \\ 5. Natural consequences*}

12. Antecedents ${ }^{\star}$

6. Comparison of behaviour*

\section{Associations*}

8. Repetition and substitution $^{*}$

13. Identity

14. Scheduled consequences

*BCT groupings represented in intervention or control group descriptions.

BCTTv1, behaviour change technique taxonomy v1.

\section{DISCUSSION}

Several BCTs were commonly used in pharmacy interventions such as 'instruction on how to perform the behaviour', 'problem solving', 'information about health consequences', 'social support (practical)' and 'social support (unspecified)'. Additionally, the control groups included a number of identifiable BCTs. Of the 93 BCTs in the BCTTv1, 31 were found across the 86 included studies, 19 of which were unique to the intervention groups.

Some of the BCTs that were frequently implemented have been found to be effective in previous research. 'Instruction on how to perform the behaviour' was identified in the intervention groups of 47 out of the 86 studies and was by far the most prominent BCT found in this review. This BCT has been effective in interventions for physical activity in inactive adults ${ }^{17}$ and diet and physical activity interventions in Type II diabetes, particularly for the reduction of haemoglobin A1c. ${ }^{18}$ This was also the most common BCT identified in control groups (10 of the 86 studies). The BCT, 'social support (unspecified)', was identified in the intervention groups of 20 out of the 86 studies. It was also the second most reported BCT in control groups, appearing in seven of the 86 studies. This BCT has been effective in physical activity interventions for people with dementia. $^{19}$

Only 31 of the 93 available BCTs from 10 of the 16 groups were represented in the 86 studies. This means that 62 BCTs and six of the 16 BCT groupings were not used (or were used but were not identifiable from the reporting) in any of the interventions. While some BCTs may not be suitable for implementation in all pharmacist interventions, it is evident that the majority $(67 \%)$ of BCTs available are not being tested at all in these types of interventions. There is, therefore, the possibility to expand the types of active ingredients in pharmacist interventions explored in future studies.
Some control groups reported BCTs that could, in theory, alter behaviour; as such, this needs to be taken into consideration when interpreting results from trials where BCTs appear in both intervention and control groups, potentially altering the apparent effect size of the intervention. If there is incomplete description of control group conditions, more BCTs might be present than reported, and as such, may affect the study outcomes. $^{121320}$

It is worth noting that 13 RCT interventions had no identifiable BCTs and yet eight of these studies reported an intervention effect. While this might suggest that these interventions contained BCTs not currently included in the BCTTv1, it is more likely that BCTs were included but not reported, thus giving a misleading picture of the intervention. Overall, reporting of the content of interventions was unclear, vague and incomplete, even with the aid of additional materials.

Several BCTs that have previously been found to be effective in behaviour change interventions were used infrequently in pharmacist interventions, suggesting that opportunities for effective intervention may be being missed. 'Action planning' was only identified in 11 studies despite growing evidence of its effectiveness. ${ }^{171821}$ 'Goal setting (behaviour)' and 'goal setting (outcome)' are effective in behaviour change interventions ${ }^{1922} 23$ but were identifiable in six and 15 studies, respectively. Similarly, 'self-monitoring (behaviour)' and 'self-monitoring (outcome)' were identifiable in five and 14 studies, respectively, despite considerable evidence of their effectiveness as BCTs. ${ }^{22-24}$ In addition, despite evidence of the effectiveness of 'demonstration of behaviour', ${ }^{18}$ 'behavioural practice/rehearsal', ${ }^{17} 18$ 'biofeedback' ${ }^{17}$ and 'feedback', ${ }^{22}$ they are seldom identifiable in the 86 RCTs. These BCTs may lack relevance to pharmacist-related activities or this finding may illustrate the need for a more explicit theory-based and evidence-based approach to designing pharmacist interventions and deciding which BCTs to include in them.

Poor reporting of interventions is a serious problem, highlighted by Hoffmann and colleagues. ${ }^{25}$ If intervention descriptions are incomplete, then effective interventions cannot be reliably implemented, replicated or synthesised, resulting in a serious waste of research effort and funding. Reporting of trials of non-pharmacological interventions has been found to lack detail and to continue to be incomplete even after contacting authors. ${ }^{26}$ Furthermore, analysis of titles and abstracts has demonstrated that the reporting of behavioural interventions has been found to be poorer than the reporting of other non-pharmacological interventions, a significant problem when this is the information typically used to identify papers for evidence synthesis. ${ }^{27}$ The studies in the current paper were assessed using the template for intervention description and replication (TIDieR) checklist ${ }^{25}$ and most were found to lack adequate intervention reporting (full results reported elsewhere ${ }^{28}$ ); however, should future 
studies use the TIDieR checklist, the quality of intervention reporting should improve.

There were both strengths and limitations to this study. A high level of agreement was achieved between coders and their application of the BCTTv1. The accuracy of the BCT coding was heavily reliant on, and therefore limited by, the information provided in the studies. While additional materials were sought from the authors, these were not always provided and/or were not always presented in sufficient detail to give an accurate description of what was being evaluated. The BCT coding study was undertaken with the 86 studies included in the interim update of the Cochrane review and not the fully updated version of the review which comprised 116 studies.

\section{CONCLUSION}

A large number of BCTs have been evaluated in pharmacy interventions. The coding of BCTs was affected by poor reporting. More transparent and comprehensive reporting of intervention and control groups would facilitate the identification of BCTs and the exploration of the association between individual BCTs and the effectiveness of interventions.

Future pharmacist interventions should be informed by the evidence of effective BCTs. Trials to evaluate these interventions should report the content of intervention and control groups using the BCTTv1 and TIDieR. This ultimately could help identify which specific behavioural interventions are most effective, specifically within the pharmacy setting.

\section{Author affiliations}

${ }^{1}$ Dental Clinical Effectiveness, NHS Education for Scotland, Dundee, UK

${ }^{2}$ Life Sciences, Brunel University London, Middlesex, UK

${ }^{3}$ Health Psychology, University of Aberdeen Institute of Applied Health Sciences, Aberdeen, UK

${ }^{4}$ Radboud Institute for Health Sciences, Radboud University Medical Centre, Nijmegen, Gelderland, The Netherlands

${ }^{5}$ Medical Statistics, University of Aberdeen, Aberdeen, UK

${ }^{6}$ Faculty of Social Sciences, University of Stirling, Stirling, UK

${ }^{7}$ Academic Primary Care, University of Aberdeen Institute of Applied Health

Sciences, Aberdeen, UK

${ }^{8}$ Strathclyde Institute of Pharmacy and Biomedical Sciences, University of Strathclyde, Glasgow, UK

\section{Twitter Christine Bond @christinebond20}

Contributors All authors, CS, MdB, MJ, MdB, NS, CM, CB and MCW, contributed to the conception and design of the work. CS and MdB coded the data, with support from MJ and MCW. CS and MCW wrote the manuscript with contribution and critical review from MdB, MJ, MdB, NS, CM and CB. Final approval of the published version was given from all authors.

Funding This work was supported by the Chief Scientist Office, grant number $\mathrm{CZH} / 4 / 1041$. MdB was also funded by the Professor Roy Weir Career Development Fellowship. MCW was funded by a Health Foundation Improvement Science Fellowship. The Health Foundation is an independent charity committed to bringing about better health and healthcare for people in the UK.

Competing interests None declared.

Patient and public involvement Patients and/or the public were not involved in the design, or conduct, or reporting, or dissemination plans of this research.

Patient consent for publication Not required.
Provenance and peer review Not commissioned; externally peer-reviewed.

Data availability statement Data are available upon reasonable request. The data sets used and analysed during the current study are available from the corresponding author on reasonable request.

Open access This is an open access article distributed in accordance with the Creative Commons Attribution Non Commercial (CC BY-NC 4.0) license, which permits others to distribute, remix, adapt, build upon this work non-commercially, and license their derivative works on different terms, provided the original work is properly cited, appropriate credit is given, any changes made indicated, and the use is non-commercial. See: http://creativecommons.org/licenses/by-nc/4.0/.

\section{ORCID iDs}

Claire Scott http://orcid.org/0000-0003-1230-6253

Mícheál de Barra http://orcid.org/0000-0003-4455-6214

\section{REFERENCES}

1 Craig P, Dieppe P, Macintyre S, et al. Developing and evaluating complex interventions: the new medical Research Council guidance. BMJ 2008;337:a1655.

2 Michie S, Richardson M, Johnston M, et al. The behavior change technique taxonomy (V1) of 93 hierarchically clustered techniques: building an international consensus for the reporting of behavior change interventions. Ann Behav Med 2013;46:81-95.

3 Michie S, Johnston M. Behavioural changeTechniques. In: Gellman MD, Turner JR, eds. Encyclopedia of behavioral medicine. New York,NY: Springer New York, 2013: 182-7.

4 Michie S, Abraham C, Eccles MP, et al. Strengthening evaluation and implementation by specifying components of behaviour change interventions: a study protocol. Implement Sci 2011;6:10.

5 Dombrowski SU, Sniehotta FF, Avenell A, et al. Identifying active ingredients in complex behavioural interventions for obese adults with obesity-related co-morbidities or additional risk factors for comorbidities: a systematic review. Health Psychol Rev 2012;6:7-32.

6 Michie S, Abraham C, Whittington C, et al. Effective techniques in healthy eating and physical activity interventions: a meta-regression. Health Psychol 2009;28:690-701.

7 Bruhn H, Bond CM, Elliott AM, et al. Pharmacist-Led management of chronic pain in primary care: results from a randomised controlled exploratory trial. BMJ Open 2013;3:e002361.

8 BLENKINSOPP A, PHELAN M, BOURNE J, et al. Extended adherence support by community pharmacists for patients with hypertension: a randomised controlled trial. Int $J$ Pharm Pract 2000;8:165-75.

9 Nkansah N, Mostovetsky O, Yu C, et al. Effect of outpatient pharmacists' non-dispensing roles on patient outcomes and prescribing patterns. Cochrane Database Syst Rev 2010;7:CD000336.

10 Avery AJ, Rodgers S, Cantrill JA, et al. A pharmacist-led information technology intervention for medication errors (pincer): a multicentre, cluster randomised, controlled trial and cost-effectiveness analysis. Lancet 2012;379:1310-9.

11 Brown TJ, Todd A, O'Malley C, et al. Community pharmacy-delivered interventions for public health priorities: a systematic review of interventions for alcohol reduction, smoking cessation and weight management, including meta-analysis for smoking cessation. BMJ Open 2016;6:e009828.

12 de Bruin M, Viechtbauer W, Hospers HJ, et al. Standard care quality determines treatment outcomes in control groups of HAARTadherence intervention studies: implications for the interpretation and comparison of intervention effects. Health Psychol 2009;28:668-74.

13 de Bruin M, Viechtbauer W, Schaalma HP, et al. Standard care impact on effects of highly active antiretroviral therapy adherence interventions: a meta-analysis of randomized controlled trials. Arch Intern Med 2010;170:240-50.

14 de Barra M, Scott CL, Scott NW, et al. Pharmacist services for non-hospitalised patients. Cochrane Database Syst Rev 2018;9:CD013102.

15 Change UCfB. Behaviour Change Technique Taxonomy v1 (BCTTv1) online training 2014. Available: https://www.bct-taxonomy.com/

16 Gwet KL. Handbook of inter-rater reliability: the definitive guide to measuring the extent of agreement among multiple raters. Advanced Analytics, LLC, 2012.

17 Howlett N, Trivedi D, Troop NA, et al. Are physical activity interventions for healthy inactive adults effective in promoting behavior change and maintenance, and which behavior change 
techniques are effective? A systematic review and meta-analysis. Trans/ Behav Med 2019;9:147-57.

18 Cradock KA, ÓLaighin G, Finucane FM, et al. Behaviour change techniques targeting both diet and physical activity in type 2 diabetes: a systematic review and meta-analysis. Int J Behav Nutr Phys Act 2017;14:18.

19 Nyman SR, Adamczewska N, Howlett N. Systematic review of behaviour change techniques to promote participation in physical activity among people with dementia. Br J Health Psychol 2018;23:148-70.

20 de Bruin M, Viechtbauer W, Eisma MC, et al. Identifying effective behavioural components of intervention and comparison group support provided in smoking cEssation (IC-SMOKE) interventions: a systematic review protocol. Syst Rev 2016;5:77.

21 Gollwitzer PM, Sheeran P. Implementation Intentions and Goal Achievement: A Meta-analysis of Effects and Processes. In: Advances in experimental social psychology. Academic Press, 2006: 69-119.

22 Carver CS, Scheier MF. Control theory: a useful conceptual framework for personality-social, clinical, and health psychology. Psychol Bull 1982;92:111-35.
23 Samdal GB, Eide GE, Barth T, et al. Effective behaviour change techniques for physical activity and healthy eating in overweight and obese adults; systematic review and meta-regression analyses. Int $J$ Behav Nutr Phys Act 2017;14:42.

24 Harkin B, Webb TL, Chang BPI, et al. Does monitoring goal progress promote goal attainment? A meta-analysis of the experimental evidence. Psychol Bull 2016;142:198-229.

25 Hoffmann TC, Glasziou PP, Boutron I, et al. Better reporting of interventions: template for intervention description and replication (TIDieR) checklist and guide. BMJ 2014;348:g1687.

26 Hoffmann TC, Erueti C, Glasziou PP. Poor description of nonpharmacological interventions: analysis of consecutive sample of randomised trials. BMJ 2013;347:f3755.

27 McCleary N, Duncan EM, Stewart F, et al. Active ingredients are reported more often for pharmacologic than non-pharmacologic interventions: an illustrative review of reporting practices in titles and Abstracts. Trials 2013;14:146.

28 de Barra M, Scott C, Johnston M, et al. Do pharmacy intervention reports adequately describe their interventions? a template for intervention description and replication analysis of reports included in a systematic review. BMJ Open 2019;9:e025511. 